Proceedings of the

International Geometry Center

Vol. 9, no. 3-4 (2016) pp. 50-58

\title{
Задача о тени и смежные задачи
}

\author{
Ю. Б. Зелинский, И. Ю. Выговская, Х. К. Дакхил
}

\begin{abstract}
We review recent results related to the shade problem, discuss unsolved problems, and give estimates of necessary and sufficient conditions for solutions of shade problem.
\end{abstract}

Аннотация. В работе дан обзор результатов, связанных с проблемой тени, полученных в исследованиях за последние полтора года. Обсуждаются нерешенные задачи и даны оценки необходимых и достаточных условий.

\section{1. ВВеДЕНИЕ}

Аксиоматический подход к определению выпуклости (говорят, что семейство множеств состоит из выпуклых множеств, если пересечение произвольного их количества принадлежит этому же семейству, [12]) позволяет назвать выпуклыми ряд экзотических классов множеств, которые не ассоциируются с привычным понятием выпуклости, например, множество всех множеств или семейство всех замкнутых подмножеств некоторого топологического пространства.

Традиционно, подмножество евклидова пространства называется въnуклым, если вместе с произвольной парой точек оно содержит и отрезок, соединяющий эти точки. Это эквивалентно связности пересечений подмножества с произвольной вещественной прямой.

Вопросы анализа часто приводят к другим семействам множеств, которые удовлетворяют аксиоме выпуклости. Ниже мы рассмотрим ряд задач, решение которых требует привлечения различных обобщений понятия выпуклости.

\footnotetext{
Ключевые слова: Евклидово пространство, сфера, шар, выпуклость, обобщенная выпуклость

УДК $513.83,517.5$
} 


\section{2. ОБОБЩЕННАЯ ВЫПУКЛОСТЬ И ЗАДАЧА О ТЕНИ}

Определение 2.1. Скажем, что множество $E \subset \mathbb{R}^{n} m$-выпукло относительно точки $x \in \mathbb{R}^{n} \backslash E$, если найдется $m$-мерная плоскость $L$, такая что $x \in L$ и $L \cap E=\varnothing$.

Определение 2.2. Скажем, что множество $E \subset \mathbb{R}^{n} m$-выпукло, если оно $m$-выпукло относительно каждой точки $x \in \mathbb{R}^{n} \backslash E$.

Оба приведенные определения удовлетворяют известной аксиоме выпуклости: пересечение каждого подсемейства таких множеств тоже удовлетворяет определению. Для произвольного множества $E \subset \mathbb{R}^{n}$ мы можем рассматривать минимальное $m$-выпуклое множество, содержащее $E$, и назвать его $m$-оболочкой множества $E$.

В работе [10] получено полное решение следующей задачи о тени, впервые рассмотренной Г. Худайбергановым [14], [7], [8].

Задача о тени. Какое минимальное число попарно непересекающихся замкнутых шаров с центрами на сфере $S^{n-1}$ в евклидовом пространстве $\mathbb{R}^{n}$ и радиуса меньшего от радиуса сферы достаточно чтобы любая прямая, проходящая через центр сферы, пересекала хотя бы один из этих шаров?

Другими словами: когда центр сферы будет принадлежать 1-выпуклой оболочке объединения шаров?

В [10] показано, что для этого необходимо и достаточно $n+1$ шара.

\section{3. СМЕЖНЫЕ ЗАДАЧИ}

Решение задачи о тени индуцировало ряд близких задач, решением которых в последние полтора года занималась группа математиков в Институте математики НАН Украины. Перечислим эти задачи.

(1) Как изменится минимальное количество шаров, если центры этих шаров не привязаны к сфере?

(2) Как изменится результат задачи (1), если вместо шара рассмотреть семейство выпуклых множеств, полученных из некоторого выпуклого множества с непустой внутренностью при помощи заданной группы преобразований?

(3) Какое минимальное число попарно непересекающихся замкнутых шаров с центрами на сфере $S^{n-1}$ и радиуса меньшего от радиуса сферы достаточно чтобы любой луч, проходящий через центр сферы, пересекал хотя бы один из этих шаров?

(4) Рассмотреть аналогичные задачи в комплексном и гиперкомплексном евклидовом пространстве. 
(5) Задача о тени не только для центра сферы, а для всех точек шара, ограниченного сферой $S^{n-1}$, которые не принадлежат объединению выбранных шаров.

(6) Задача о тени для точек сферы $S^{n-1}$, которые не принадлежат объединению выбранных шаров, относительно прямых касательных к сфере.

(7) Задача о тени для шаров равного радиуса.

(8) Исследовать задачу при изменении группы преобразований.

(9) Задача о тени для семейств выпуклых множеств, которые могут пересекаться между собой.

\section{4. ОСНОВНЫЕ РЕЗУЛЬТАТЫ}

Рассмотрим состояние полученных ответов на перечисленные задачи. Следующий результат дает полный ответ на задачу (1).

Теорема 4.1. [9] Для того чтобы выбранная точка в клидовом пространстве при $n \geq 2$ принадлежсала 1-выпуклой оболочке семейства попарно непересекающихся открытых (замкнутых) шаров, которые данную точку не содержат, необходимо и достаточно $n$ шаров.

Задача о тени при $n=3$, когда центры шаров расположены на эллипсоиде вращения, рассмотрена в [13]. Показано, что если отношение большей оси эллипса к меньшей больше или равно $2 \sqrt{2}$, то для создания тени достаточно трех шаров.

Полный ответ на задачу (2) содержится в следующем результате.

Теорема 4.2. [9] Для того чтобы выбранная точка в $n$-мерном евклидовом пространстве при $n \geq 2$ принадлежала 1-оболочке семейства попарно непересекающихся замкнутых множеств, полученного из заданного выпуклого множества с непустой внутренностъю при помощи группы преобразований, состоящей из движений и гомотетий, необходимо и достаточно п элементов семейства.

Если группу преобразований уменьшить, то количество элементов семейства, необходимых для создания тени в центре сферы может увеличиться. Однако, в [11] показано, что результат не изменится, если из группы движений исключить повороты.

Определение 4.3. Скажем, что множество $E \subset \mathbb{R}^{n} m$-nолувыпукло относительно точки $x \in \mathbb{R}^{n} \backslash E$, если найдется $m$-мерная полуплоскость $P$, такая что $x \in P$ и $P \cap E=\varnothing$. 
Определение 4.4. Скажем, что множество $E \subset \mathbb{R}^{n} m$-nолувыпукло, если оно $m$-полувыпукло относительно каждой точки $x \in \mathbb{R}^{n} \backslash E$.

Легко убедиться, что и эти определения удовлетворяют аксиоме выпуклости, и мы тоже можем строить $m$-полувыпуклые оболочки множеств согласно этим определениям.

Для задачи (3) получена оценка сверху в размерности три. Задача точной оценки остается открытой, неизвестны даже оценки сверху в размерностях больше трех.

Теорема 4.5. [4] Для того чтобы иентр двумерной сберы в трехмерном евклидовом пространстве принадлежал 1-полувыпуклой оболочке семейства открытых (соотв. замкнутых) шаров радиуса не превышающего (соотв. менъшего) радиуса сферь и с иентрами на сфере достаточно десяти шаров.

Если объединить вопросы задач (1) и (3), то получен точный результат.

Теорема 4.6. [9] Для того чтобы выбранная точка в $n$-мерном евклидовом пространстве при $n \geq 2$ принадлежала 1-полувыпуклой оболочке семейства попарно непересекающихся открытых (замкнутых) шаров, которье данную точку не содержат, необходимо и достаточно $n+1$ uара.

Следующий результат показывает, что в предыдущей теореме можно заменить шары выпуклыми телами с непустой внутренностью.

Теорема 4.7. [9] Для того чтобы выбранная точка в клидовом пространстве при $n \geq 2$ принадлежала 1-полувыпуклой оболочке семейства попарно непересекаюшихся замкнутых множеств, полученного из заданного выпуклого множества с непустой внутренностъю при помощи группь преобразований, состоящей из движений и гомотетий, необходимо и достаточно $n+1$ элемента семейства.

Но, если уменьшать группу допустимых преобразований, то количество необходимых для создания тени множеств увеличивается. Критическим модельным множеством будет прямоугольный параллелепипед.

Частичный ответ на задачу (8) дает следующий результат.

Теорема 4.8. [11] Для того чтобы выбранная точка в $n$-мерном евклидовом пространстве при $n \geq 2$ принадлежала 1-полувыпуклой оболочке семейства попарно непересекаюшихся замкнутых множеств, 
полученного из заданного выпуклого множества с непустой внутренностью при помощи группы преобразований, состоящей из параллельных переносов и гомотетий, необходимо и достаточно $2 n$ элементов семейства.

В случае комплексного или гиперкомплексного евклидового пространства можно рассмотреть аналогичные задачи относительно пересечений семейств, соответственно, комплексными или гиперкомплексными плоскостями.

Определение 4.9. Скажем, что множество $E \subset \mathbb{C}^{n}\left(\right.$ соотв. $\left.E \subset \mathbb{H}^{n}\right)$ $m$-комплексно (соотв. $m$-гиперкомплексно) выпукло относительно точки $z \in \mathbb{C}^{n} \backslash E$ (соотв. $z \in \mathbb{H}^{n} \backslash E$ ), если найдется $m$-мерная комплексная (гиперкомплексная) плоскость $L$, такая что $z \in L$ и $L \cap E=\varnothing$. Скажем, что множество $E \subset \mathbb{C}^{n}$ (соотв. $E \subset \mathbb{H}^{n}$ ) $m$-комплексно (соотв. $m$ гиперкомплексно) выпукло, если оно $m$-комплексно (соотв. $m$-гиперкомплексно) выпукло относительно каждой точки $z \in \mathbb{C}^{n} \backslash E$ (соотв. $\left.E \subset \mathbb{H}^{n}\right)$.

Для задачи (4) с шарами, центры которых находятся на сфере, получена точная оценка при $n=2$ и оценка сверху при $n>2$.

Теорема 4.10. [4] Для того чтобъ начало координат в двумерном комплексном (соотв. гиперкомплексном) евклидовом пространстве $\mathbb{C}^{2}$ (соотв. $\mathbb{H}^{2}$ ) принадлежало 1-комплексной (соотв. 1-гиперкомплексной) оболочке семейства попарно непересекающихся открытых (соотв. замкнутых) шаров с иентрами на единичной сфере $S^{3}$ (соотв. $S^{7}$ ), которые начало координат не содержст, необходимо и достаточно двух шаров.

Теорема 4.11. [5] Для того чтобы начало координат в $n$-мерном комплексном (соотв. гиперкомплексном) евклидовом пространстве $\mathbb{C}^{n}$ (соотв. $\left.\mathbb{H}^{n}\right), n>2$ принадлежсало 1-комплексной (соотв. 1-гиперкомплексной) оболочке семейства попарно непересекающихся открытых (соотв. замкнутых) шаров с иентрами на единичной сфере $S^{2 n-1} \subset \mathbb{C}^{n}$ (соотв. $\left.S^{4 n-1} \subset \mathbb{H}^{n}\right)$, и которые начало координат не содержсат, достаточно $2 n$ (соотв. $4 n-1$ ) шаров.

Также в (гипер)комплексном случае получаем полный аналог теоремы 4.1, если центры шаров не привязаны к сфере.

Теорема 4.12. [6] Для того чтобы начало координат в $n$-мерном комплексном (соотв. гиперкомплексном) евклидовом пространстве $\mathbb{C}^{n}$ 
(соотв. $\left.\mathbb{H}^{n}\right), n>2$ принадлежсало 1-комплексной (соотв. 1-гиперкомплексной) оболочке семейства попарно непересекающихся открытых (соотв. замкнутых) шаров, которые начало координат не содержсат, достаточно $n$ шаров.

Задача (5) весьма сложная. Здесь кроме почти очевидного факта, что при $n=2$ достаточно трех шаров размещенных в вершинах равностороннего треугольника, радиус которых равен половине высоты треугольника, больше ничего неизвестно.

Определение 4.13. Скажем, что семейство множеств $\mathfrak{J}=\left\{F_{\alpha}\right\}$ задает тень касательную к многообразию $M$ в точке $x \in M$, если каждая прямая, касательная к многообразию $M$ в точке $x \in M \backslash \bigcup_{\alpha} F_{\alpha}$, имеет непустое пересечение хотя бы с одним множеством $F_{\alpha}$, принадлежащим к семейству $\mathfrak{J}$.

Задачу (6) при $n=3$ можно сформулировать следующим образом. Найти минимальное количество попарно непересекающихся шаров с центрами на сфере $S^{2} \subset \mathbb{R}^{3}$, которые обеспечат тень касательную к cфере $S^{2}$ во всех точках $x \in S^{2} \backslash \cup_{\alpha} F_{\alpha}$.

Лемма 4.14. Рассмотрим равносторонний треугольник $A B C$ в евклидовой плоскости $\mathbb{R}^{2}$. Если ми выберем три круга $B_{i}, i=1,2,3$ с центрами в вершинах этого треугольника и радиуса равного половине высоты треугольника, то каждая прямая через произвольную точку $x \in\left(\cup_{i=1}^{3} B_{i}\right)^{*} \backslash \bigcup_{i=1}^{3} B_{i}$, где $\left(\cup_{i=1}^{3} B_{i}\right)^{*}$ - выпуклая оболочка множества $\bigcup_{i=1}^{3} B_{i}$, пересекается не менее чем с одним из выбранных кругов.

Отметим, что окружность, описанная вокруг этого треугольника, лежит в выпуклой оболочке этих трех шаров. Лемма решает задачу (4) при $n=2$. Этот результат показывает, что в трехмерном случае также для произвольной точки сферы можно выбрать три попарно касающиеся шара, которые обеспечат тень во всех точках криволинейного треугольника, вырезанного на сфере этими шарами. Однако, как показывает следующий пример, согласование такой конструкции для всей сферы требует дополнительных рассуждений.

Пример 1. Существует набор из 14 открытых (замкнутых) попарно непересекающихся шаров с центрами на сфере $S^{2} \subset \mathbb{R}^{3}$, который не обеспечивает тень касательную к сфере $S^{2}$ в каждой точке $x \in$ $S^{2} \backslash \bigcup_{i=1}^{14} F_{i}$. 
Не нарушая общности можно предполагать, что выбрана сфера $S^{2}$ с центром в начале координат радиуса единица. Впишем в эту сферу куб с вершинами в точках $(x= \pm 1 / \sqrt{3}, y= \pm 1 / \sqrt{3}, z= \pm 1 / \sqrt{3})$. Длина ребра куба равна $a=2 / \sqrt{3}$. Теперь выберем восемь открытых шаров с центрами в вершинах куба и радиуса равного половине ребра куба $r=1 / \sqrt{3} \approx 0.577$. Добавим к этому семейству шесть новых открытых шара с центрами в точках пересечения лучей, выходящих из начала координат и проходящих через центр грани куба, со сферой $S^{2}$. Радиусы этих шаров равны

$$
r_{1}=\sqrt{2-2 / \sqrt{3}}-1 / \sqrt{3}
$$

Каждый из них касается ровно четырех ранее выбранных шаров. Выберем двумерную плоскость, заданную уравнением $z=0$, проходящую через экваториальную окружность сферы, которая проходит через четыре центра

$$
(1,0,0), \quad(0,1,0), \quad(-1,0,0), \quad(0,-1,0)
$$

шаров меньшего радиуса $r_{1} \approx 0.342$. Поскольку $1<\sqrt{2} / 2+r_{1} \approx 1,049$, то прямая линия, касательная к экваториальной окружности в точке $(\sqrt{2}, \sqrt{2}, 0)$, пересекает шары меньшего радиуса.

Для плоскости $x+y=2 \sqrt{2}$, касательной к сфере в точке $(\sqrt{2}, \sqrt{2}, 0)$, в пересечении с системой шаров получаем две пары окружностей, каждая пара из которых симметрична относительно точки $(\sqrt{2}, \sqrt{2}, 0)$, а их центры находятся на паре перпендикулярных прямых.

Радиусы этих окружностей легко вычисляются. Имеем две окружности радиуса $r_{2} \approx 0.547$. Их центры удалены от точки пересечения прямых на расстояние $l_{2} \approx 0.577$. Другая пара окружностей радиуса $r_{3} \approx 0.1766$ имеет центры, удаленные от точки пересечения прямых на расстояние $l_{3} \approx 0.707$. Подсчитывая синусы углов, под которыми их полуокружности видны из точки $(\sqrt{2}, \sqrt{2}, 0)$ (совпадающей с точкой пересечения прямых), получим значения 0.948 и 0.249 , соответственно. Им соответствуют дуги в меньше чем $71.5^{\circ}$ и $14.5^{\circ}$ в градусном измерении. Поскольку их сумма меньше $90^{\circ}$, приходим к заключению, что существует прямая в касательной плоскости, проходящая через точку $(\sqrt{2}, \sqrt{2}, 0)$, которая не пересекает ни одну из выделенных окружностей.

Сохраним центры семейства шаров, а радиусы шаров выберем равными половине расстояния от вершин куба к центрам меньших шаров $r \approx 0.4597$. Аналогично предыдущим рассуждениям в сечении с плоскостью $x+y=2 \sqrt{2}$ получим две пары окружностей радиусов $r_{4} \approx 0.4215$ и $r_{5} \approx 0.354$, соответственно. Подсчитывая синусы углов, под которыми их полуокружности видны из точки $(\sqrt{2}, \sqrt{2}, 0)$, получим значения 0.731 и 0,5 . Им соответствуют дуги в меньше чем $47^{\circ}$ и $30^{\circ}$. Поскольку 
их сумма также меньше $90^{\circ}$, приходим к заключению, что существует прямая в касательной плоскости, проходящая через точку $(\sqrt{2}, \sqrt{2}, 0)$, которая не пересекает ни одну из выделенных окружностей. Кстати, во втором случае зазор между шарами увеличивается.

Построенный набор дает оценку снизу необходимого количества шаров. Оценка сверху остается открытой.

В следующем примере рассмотрим попытку решить задачу о тени и задачу (3) для шаров равного радиуса.

Пример 2. Впишем в сферу правильный икосаэдр. Предположим, что сфера $S^{2}$ единичного радиуса. Тогда, [2], длина ребра икосаэдра равна

$$
a=2 \sqrt{2 /(5+\sqrt{5})} .
$$

Выберем 12 шаров радиуса $r=a / 2$ в вершинах икосаэдра. Предположим, что этот набор шаров решает задачу. Как показано в [10], каждый такой шар задает конус прямых из центра сферы, который вырезает на сфере окружность радиуса $a / 2 \approx 0.52573$. Если в сферу вписать правильный додекаэдр, то длина ребра додекаэдра равна $a_{1}=4 /[\sqrt{3} \sqrt{1+\sqrt{5}}] \approx 0.7136$, [1]. Радиус окружности описанной вокруг правильного пятиугольника (грани додекаэдра) найдем из формулы $t=R \sqrt{(5-\sqrt{5}) / 2}$, где $t$ - длина ребра додекаэдра [3]. Следовательно,

$$
R=\frac{4 \sqrt{2 / 3}}{\sqrt{1+\sqrt{5}} \cdot \sqrt{5-\sqrt{5}}} \approx 0.607 .
$$

Поскольку этот радиус больше $a / 2$, то выбранных 12 шаров недостаточно для создания тени относительно луча из центра сферы.

О задачах (6) и (7) результатов почти нет. Интересны любые оценки.

Несложно показать, что задача (9) эквивалентна задаче (1) для 1выпуклости и задаче (3) для 1-полувыпуклости.

\section{ЛитерАТУРА}

[1] http://ru.wikipedia.org/wiki/Додекаэдр.

[2] http://ru.wikipedia.org/wiki/Икосаэдр.

[3] http://ru.wikipedia.org/wiki/Правильный_пятиугольник.

[4] Y. B. Zelinskii. Generalized convex envelopes of sets and the problem of shadow. Journal of Mathematical Sciences, 211(5):710-717, 2015.

[5] Y. B. Zelinskii. Problem of shadow (complex case). Advances in Mathematics: Scientific Journal, 5(1):1-5, 2016.

[6] Y. B. Zelinskii. The problem of the shadows. Bulletin de la societé des sci. et letters de Lódź, 66(1):37-42, 2016. 
[7] Ю. Б. Зелинский. Многозначные отображения в анализе. Киев: Наукова думка, 1993.

[8] Ю. Б. Зелинский. Выпуклость. Избранные главы, volume 92. Праці Інституту математики НАН України, 2012.

[9] Ю. Б. Зелинский. Задача о тени для семейства множеств. Збірник праць Інституту математики НАН України, 12(4):197-204, 2015.

[10] Ю. Б. Зелинський, Выговская И. Ю., М. В. Стефанчук. Обобщённо выпуклые множества и задача о тени. Украӥнський математичний журнал, 67(12):1659$1666,2015$.

[11] Ю. Б. Зелінський, М. В. Стефанчук. Узагальнення задачі про тінь. Украйнсъкий математичний журнал, 68(6):657-662, 2016.

[12] В. П. Солтан. Введение в аксиоматическую теорию выпуклости. Кишинев: Штиинца, 1984.

[13] М. В. Ткачук, Т. М. Осипчук. Задача о тени для эллипсоида вращения. Збірник праць Інституту математики НАН Украӥни, 12(3):243-250, 2015.

[14] Г. Худайберганов. Об однородно-полиномиально выпуклой оболочке объединения шаров. Рукопись деп. в ВИНИТИ 21.02.1982 г. № 1772 - 85 Деп.

Поступила в редакичю 18 июля 2016, принята к печати 15 сентября 2016.

Зелинский Юрий Борисович

ИНСТИТУТ МАТЕМАТИКИ НАН УКРАИНЫ, КИЕВ, УКРАИНА

Email: zel@imath.kiev.ua

Выговская Ирина Юрьевна

ИНСТИТУТ МАТЕМАТИКИ НАН УКРАИНЫ, КИЕВ, УКРАИНА

Email: vkirinata@gmail.com

Дакхил Хайджаа Кхудхаир

КГУ ИМ. Т. ШЕВЧЕНКА, КИЕВ, УКРАИНА

Email: moonm5385@gmail.com 